\title{
On Solution of the Problem of Bending of Orthotropic Plates on the Basis of Bimoment Theory
}

\author{
Makhamatali K. Usarov \\ Institute of Seismic Stability of Structures, Academy of Sciences of Uzbekistan, Tashkent, Uzbekistan \\ Email: umakhamatali@mail.ru
}

Received 15 April 2015; accepted 8 May 2015; published 13 May 2015

Copyright (C) 2015 by author and Scientific Research Publishing Inc.

This work is licensed under the Creative Commons Attribution International License (CC BY).

http://creativecommons.org/licenses/by/4.0/

c) (i) Open Access

\section{Abstract}

This paper is devoted to the development of new theory of orthotropic thick plates with account of internal forces, moments and bimoments. An equation of motion of plates is described by two systems with nine equations each. Boundary conditions depended on displacements, forces, moments and bimoments are given. An exact solution of the bending of thick plate under the effect of sine load is built. Numerical results for maximal values of displacements and stresses of the plate are obtained.

\section{Keywords}

Plate, Orthotropy, Isotropy, Force, Moment, Bimoment, Equation of Motion, Exact Solution

\section{Introduction}

Specified theories of plates are widely used in analysis of structure elements. Review and general technique for constructing a specified theory can be found in [1] [2]. In spatial case of bending and vibrations along the thickness of the plate, the displacements vary according to nonlinear law, and classical theories of plates and shells become unacceptable. In general case the field of displacement of thick plates does not obey to any simplifying hypotheses. It is necessary to take into account all the components of the tensor of stress and strain:

$\sigma_{i j}, \varepsilon_{i j},(i, j=1,3)$; by them we will introduce tensile and crosscutting forces, bending and torsion moments and the concept of bimoments [3], generated due to nonlinear law of distribution of displacements in cross-sections of the plate.

This article briefly describes a method of constructing a theory of plates with bimoments. Determinant correlations of forces, moments, bimoments and the equations of motion in relation to these types of force factors are 
given.

\section{Statement of the Problem}

Consider orthotropic thick plate of constant thickness $H=2 h$ and dimensions $a, b$ in plan. Introduce the signs: $E_{1}, E_{2}, E_{3}$ - elasticity modulus and $G_{12}, G_{13}, G_{23}$-shear modulus; $v_{12}, v_{13}, v_{23}$-Poisson's ratio of plate material.

Introduce Cartesian system of coordinates $x_{1}, x_{2}$ and $z$. Axis $o z$ is directed vertically downwards. Let distributed surface normal and tangential loads are applied to the lower and the upper face surfaces of the plate $z=h$ and $z=-h$. Normal loads in $o z$ axis we will designate as $q_{3}^{(+)}, q_{3}^{(-)}$, tangential loads in direction $o x_{1}, o x_{2}-q_{k}^{(+)}, q_{k}^{(-)},(k=\overline{1,2})$. Vlasov B. F. [4] has built an exact analytical solution of this problem in trigonometric series.

Components of the vector of displacement are determined by the functions of three spatial coordinates and time $u_{1}\left(x_{1}, x_{2}, z, t\right), u_{2}\left(x_{1}, x_{2}, z, t\right), u_{3}\left(x_{1}, x_{2}, z, t\right)$. Components of the tensor of strain are determined by Cauchy correlation. The plate is considered as a three-dimensional body, its material obeying Hooke's generalized law:

$$
\begin{aligned}
& \sigma_{11}=E_{11} \varepsilon_{11}+E_{12} \varepsilon_{22}+E_{13} \varepsilon_{33}, \quad \sigma_{22}=E_{21} \varepsilon_{11}+E_{22} \varepsilon_{22}+E_{23} \varepsilon_{33} \text {, } \\
& \sigma_{33}=E_{31} \varepsilon_{11}+E_{32} \varepsilon_{22}+E_{33} \varepsilon_{33}, \quad \sigma_{12}=2 G_{12} \varepsilon_{12}, \quad \sigma_{13}=2 G_{13} \varepsilon_{13}, \quad \sigma_{23}=2 G_{23} \varepsilon_{23} \text {, }
\end{aligned}
$$

where $E_{11}, E_{12}, \cdots, E_{33}$-are elastic constants, defined by Poisson's ratio and elasticity modulus in the form:

$$
\begin{gathered}
E_{11}=E_{1} g_{11}, E_{22}=E_{2} g_{22}, E_{33}=E_{3} g_{33}, \\
E_{12}=E_{21}=E_{1} g_{12}=E_{2} g_{21}, E_{13}=E_{31}=E_{1} g_{13}=E_{3} g_{31}, E_{23}=E_{32}=E_{2} g_{23}=E_{3} g_{32}, \\
g_{11}=\frac{1-v_{23} v_{32}}{1-\mu^{2}}, g_{22}=\frac{1-v_{13} v_{31}}{1-\mu^{2}}, g_{33}=\frac{1-v_{12} v_{21}}{1-\mu^{2}}, \\
g_{12}=g_{21}=\frac{v_{12}+v_{13} v_{32}}{1-\mu^{2}}=\frac{v_{21}+v_{31} v_{23}}{1-\mu^{2}}, \quad g_{13}=g_{31}=\frac{v_{13}+v_{21} v_{32}}{1-\mu^{2}}=\frac{v_{31}+v_{12} v_{23}}{1-\mu^{2}}, \\
g_{23}=g_{32}=\frac{v_{23}+v_{13} v_{12}}{1-\mu^{2}}=\frac{v_{32}+v_{31} v_{21}}{1-\mu^{2}}, \mu^{2}=v_{12} v_{21}+v_{23} v_{32}+v_{13} v_{31}+2 v_{12} v_{23} v_{31} .
\end{gathered}
$$

As an equation of motion of the plate we will use three-dimensional equations of motion of the theory of elasticity:

$$
\frac{\partial \sigma_{i 1}}{\partial x_{1}}+\frac{\partial \sigma_{i 2}}{\partial x_{2}}+\frac{\partial \sigma_{i 3}}{\partial z}=\rho \ddot{u}_{i},(i=1,3) .
$$

here $\rho$-is a density of plate material.

Boundary conditions on the lower and the upper surfaces $z=h$ and $z=-h$ have the form:

$$
\begin{aligned}
& \sigma_{33}=q_{3}^{(+)}, \quad \sigma_{31}=q_{1}^{(+)}, \sigma_{32}=q_{2}^{(+)}, \text {at } z=h ; \\
& \sigma_{33}=q_{3}^{(-)}, \quad \sigma_{31}=q_{1}^{(-)}, \sigma_{32}=q_{2}^{(-)}, \quad \text { at } z=-h \text {. }
\end{aligned}
$$

\section{Method of Solution}

Methods of constructing a bimoment theory of plates is based on displacements expansions in infinite series, Hooke's generalized law (1), three-dimensional equations of the theory of elasticity (2) and boundary conditions on face surfaces (3). Components of the vector of displacements are expanded in Macloren's series in the form [3]-[7]:

$$
u_{k}=B_{0}^{(k)}+B_{1}^{(k)} \frac{z}{h}+B_{2}^{(k)}\left(\frac{z}{h}\right)^{2}+B_{3}^{(k)}\left(\frac{z}{h}\right)^{3}+\cdots+B_{m}^{(k)}\left(\frac{z}{h}\right)^{m}+\cdots, \quad(k=1,2),
$$




$$
u_{3}=A_{0}+A_{1} \frac{z}{h}+A_{2}\left(\frac{z}{h}\right)^{2}+A_{3}\left(\frac{z}{h}\right)^{3}+\cdots+A_{m}\left(\frac{z}{h}\right)^{m}+\cdots
$$

here $B_{m}^{(k)}, A_{m}$-are unknown functions of two spatial coordinates and time:

$$
B_{m}^{(k)}=B_{m}^{(k)}\left(x_{1}, x_{2}, t\right)=\frac{h^{m}}{m !}\left(\frac{\partial^{m} u_{k}}{\partial z^{m}}\right)_{z=0},(k=1,2), \quad A_{m}=A_{m}\left(x_{1}, x_{2}, t\right)=\frac{h^{m}}{m !}\left(\frac{\partial^{m} u_{3}}{\partial z^{m}}\right)_{z=0} .
$$

Offered bimoment theory of plates [5]-[8] is described by two unrelated problems, each of which is formulated on the basis of nine two-dimensional equations with corresponding boundary conditions. It should be noted that proposed bimoment theory of plates presents a two-dimensional theory of elastic orthotropic layer, which is deformed in general three-dimensional form.

The first problem is described by two equations relative to longitudinal and tangential forces, by four additionally constructed equations in relation to bimoments and three equations obtained from the boundary conditions (3) on the basis of expansion (4). The forces, moments and bimoments of the plate are determined by nine unknown kinematic functions from relationships [5]-[8]:

$$
\begin{gathered}
\bar{u}_{k}=\frac{u_{k}^{(+)}+u_{k}^{(-)}}{2}, \quad \bar{\psi}_{k}=\frac{1}{2 h} \int_{-h}^{h} u_{k} \mathrm{~d} z, \quad \bar{\beta}_{k}=\frac{1}{2 h^{3}} \int_{-h}^{h} u_{k} z^{2} \mathrm{~d} z, \quad(k=1,2), \\
\bar{W}=\frac{u_{3}^{(+)}-u_{3}^{(-)}}{2}, \quad \bar{r}=\frac{1}{2 h^{2}} \int_{-h}^{h} u_{3} z \mathrm{~d} z, \quad \bar{\gamma}=\frac{1}{2 h^{4}} \int_{-h}^{h} u_{3} z^{3} \mathrm{~d} z .
\end{gathered}
$$

We will get the equations of equilibrium relative to longitudinal and tangential forces by integrating two first equations of the theory of elasticity in coordinate $z$ (2):

$$
\frac{\partial N_{11}}{\partial x_{1}}+\frac{\partial N_{12}}{\partial x_{2}}+2 \bar{q}_{1}=\rho H \ddot{\bar{\psi}}, \quad \frac{\partial N_{21}}{\partial x_{1}}+\frac{\partial N_{22}}{\partial x_{2}}+2 \bar{q}_{2}=\rho H \ddot{\bar{\psi}}_{2},
$$

where $N_{11}, N_{12}, N_{22}$-are longitudinal and tangential forces determined from relationships

$$
\begin{gathered}
N_{11}=\int_{-h}^{h} \sigma_{11} \mathrm{~d} z=E_{11} H \frac{\partial \bar{\psi}_{1}}{\partial x_{1}}+E_{12} H \frac{\partial \bar{\psi}_{2}}{\partial x_{2}}+2 E_{13} \bar{W}, \\
N_{22}=\int_{-h}^{h} \sigma_{22} \mathrm{~d} z=E_{12} H \frac{\partial \bar{\psi}_{1}}{\partial x_{1}}+E_{22} H \frac{\partial \bar{\psi}_{2}}{\partial x_{2}}+2 E_{23} \bar{W}, \\
N_{12}=N_{21}=\int_{-h}^{h} \sigma_{12} \mathrm{~d} z=G_{12}\left(H \frac{\partial \bar{\psi}_{2}}{\partial x_{1}}+H \frac{\partial \bar{\psi}_{1}}{\partial x_{2}}\right),
\end{gathered}
$$

$\bar{q}_{k}=\frac{q_{k}^{(+)}-q_{k}^{(-)}}{2},(k=1,2), \bar{q}_{3}=\frac{q_{3}^{(+)}+q_{3}^{(-)}}{2}$-terms of equations with external load.

On the basis of force expression (7) two Equations (6) include three unknown functions $\bar{\psi}_{1}, \bar{\psi}_{2}, \bar{r}$. To derive additional equations we will introduce bimoments, generated in tension and cross compression of the plate. Longitudinal and tangential bimoments are determined by expressions: $T_{11}, T_{22}, T_{12}$ :

$$
\begin{gathered}
T_{11}=\frac{1}{h^{2}} \int_{-h}^{h} \sigma_{11} z^{2} \mathrm{~d} z=H\left(E_{11} \frac{\partial \bar{\beta}_{1}}{\partial x_{1}}+E_{12} \frac{\partial \bar{\beta}_{2}}{\partial x_{2}}+E_{13} \frac{2 \bar{W}-4 \bar{r}}{H}\right), \\
T_{22}=\frac{1}{h^{2}} \int_{-h}^{h} \sigma_{22} z^{2} \mathrm{~d} z=H\left(E_{12} \frac{\partial \bar{\beta}_{1}}{\partial x_{1}}+E_{22} \frac{\partial \bar{\beta}_{2}}{\partial x_{2}}+E_{23} \frac{2 \bar{W}-4 \bar{r}}{H}\right), \\
T_{12}=T_{21}=\frac{1}{h^{2}} \int_{-h}^{h} \sigma_{12} z^{2} \mathrm{~d} z=H G_{12}\left(\frac{\partial \bar{\beta}_{2}}{\partial x_{1}}+\frac{\partial \bar{\beta}_{1}}{\partial x_{2}}\right) .
\end{gathered}
$$

Introduce intensities of transversal bimoments $\bar{p}_{13}, \bar{p}_{23}$ and $\bar{\tau}_{13}, \bar{\tau}_{23}$ on tangential stresses $\sigma_{13}, \sigma_{23}$ : 


$$
\begin{gathered}
\bar{p}_{k 3}=\frac{1}{2 h^{2}} \int_{-h}^{h} \sigma_{k 3} z \mathrm{~d} z=G_{k 3}\left(\frac{\partial \bar{r}}{\partial x_{k}}+\frac{2\left(\bar{u}_{k}-\bar{\psi}_{k}\right)}{H}\right), \quad(k=1,2), \\
\bar{\tau}_{k 3}=\frac{1}{2 h^{4}} \int_{-h}^{h} \sigma_{k 3} z^{3} \mathrm{~d} z=G_{k 3}\left(\frac{\partial \bar{\gamma}}{\partial x_{k}}+\frac{2\left(\bar{u}_{k}-3 \bar{\beta}_{k}\right)}{H}\right), \quad(k=1,2) .
\end{gathered}
$$

Introduce intensities of normal bimoments $\bar{p}_{33}$ and $\bar{\tau}_{33}$ on normal stress $\sigma_{33}$ in the form of relations:

$$
\begin{gathered}
\bar{p}_{33}=\frac{1}{2 h} \int_{-h}^{h} \sigma_{33} \mathrm{~d} z=E_{31} \frac{\partial \bar{\psi}_{1}}{\partial x_{1}}+E_{32} \frac{\partial \bar{\psi}_{2}}{\partial x_{2}}+E_{33} \frac{2 \bar{W}}{H}, \\
\bar{\tau}_{33}=\frac{1}{2 h^{3}} \int_{-h}^{h} \sigma_{33} z^{2} \mathrm{~d} z=E_{31} \frac{\partial \bar{\beta}_{1}}{\partial x_{1}}+E_{32} \frac{\partial \bar{\beta}_{2}}{\partial x_{2}}+E_{33} \frac{2 \bar{W}-4 \bar{r}}{H} .
\end{gathered}
$$

Equations in relation to longitudinal and transversal bimoments, acting in plate plane are obtained in the form:

$$
\begin{array}{cl}
\frac{\partial T_{11}}{\partial x_{1}}+\frac{\partial T_{12}}{\partial x_{2}}-4 \bar{p}_{13}+2 \bar{q}_{1}=\rho H \ddot{\bar{\beta}}, & \frac{\partial T_{12}}{\partial x_{1}}+\frac{\partial T_{22}}{\partial x_{2}}-4 \bar{p}_{23}+2 \bar{q}_{2}=\rho H \ddot{\bar{\beta}_{2}}, \\
\frac{\partial \bar{p}_{13}}{\partial x_{1}}+\frac{\partial \bar{p}_{23}}{\partial x_{2}}-\frac{2 \bar{p}_{33}}{H}+\frac{2 \bar{q}_{3}}{H}=\rho \ddot{\bar{r}}, & \frac{\partial \bar{\tau}_{13}}{\partial x_{1}}+\frac{\partial \bar{\tau}_{23}}{\partial x_{2}}-\frac{6 \bar{\tau}_{33}}{H}+\frac{2 \bar{q}_{3}}{H}=\rho \ddot{\bar{\gamma}} .
\end{array}
$$

By using series (4) and Formulas (5) are obtained expressions for series' (4) coefficients $B_{2 i}^{(1)}, B_{2 i}^{(2)}, A_{2 i+1},(i=0,1,2)$ via $\bar{u}_{k}, \bar{\psi}_{k}, \bar{\beta}_{k}$ functions and boundary conditions (3) let represent as next equations:

$$
\begin{gathered}
\bar{u}_{k}=\frac{1}{4}\left(21 \bar{\beta}_{k}-3 \bar{\psi}_{k}\right)-\frac{1}{20} H \frac{\partial \bar{W}}{\partial x_{k}}+\frac{1}{20} \frac{H \bar{q}_{k}}{G_{k 3}}, \quad(k=1,2), \\
\bar{W}=\frac{1}{2}(21 \bar{\gamma}-7 \bar{r})-\frac{1}{30} H\left(\frac{E_{31}}{E_{33}} \frac{\partial \bar{u}_{1}}{\partial x_{1}}+\frac{E_{32}}{E_{33}} \frac{\partial \bar{u}_{2}}{\partial x_{2}}\right)+\frac{H \bar{q}_{3}}{30 E_{33}} .
\end{gathered}
$$

Equations (6), (11), (12) and (13) make a combined system of differential equations of motion, which consists of nine equations relative to nine unknown functions: $\bar{\psi}_{1}, \bar{\psi}_{2}, \bar{\beta}_{1}, \bar{\beta}_{1}, \bar{u}_{1}, \bar{u}_{2}, \bar{r}, \bar{\gamma}, \bar{W}$.

The second problem is described by two equations of moments, one equation of crosscutting forces, three equations of bimoments and three equations, obtained from boundary conditions (3) on the bases of expansion (4). Here forces, moments and bimoments are determined relative to nine unknown kinematic functions in the form [5]-[8]:

$$
\begin{gathered}
\tilde{W}=\frac{u_{3}^{(+)}+u_{3}^{(-)}}{2}, \quad \tilde{r}=\frac{1}{2 h} \int_{-h}^{h} u_{3} \mathrm{~d} z, \quad \tilde{\gamma}=\frac{1}{2 h^{3}} \int_{-h}^{h} u_{3} z^{2} \mathrm{~d} z, \\
\tilde{u}_{k}=\frac{u_{k}^{(+)}-u_{k}^{(-)}}{2}, \quad \tilde{\psi}_{k}=\frac{1}{2 h^{2}} \int_{-h}^{h} u_{k} z \mathrm{~d} z, \quad \tilde{\beta}_{k}=\frac{1}{2 h^{4}} \int_{-h}^{h} u_{k} z^{3} \mathrm{~d} z, \quad(k=1,2) .
\end{gathered}
$$

The first three of these equations are the ones relative to bending, torsion moments and an equation relative to crosscutting forces, the rest three equations are derived in relation to bimoments.

Multiplying the first and the second equations of the theory of elasticity by coordinate $z$ and integrating it by $z$, we will obtain an equation of equilibrium in moments and forces:

$$
\frac{\partial M_{11}}{\partial x_{1}}+\frac{\partial M_{12}}{\partial x_{2}}-Q_{13}+H \tilde{q}_{1}=\frac{H^{2}}{2} \rho \ddot{\tilde{\psi}}_{1}, \quad \frac{\partial M_{21}}{\partial x_{1}}+\frac{\partial M_{22}}{\partial x_{2}}-Q_{23}+H \tilde{q}_{2}=\frac{H^{2}}{2} \rho \ddot{\tilde{\psi}}_{2} .
$$

Integrating the third equation of the theory of elasticity by coordinate $z$ (2), we will obtain an equation of equilibrium in forces: 


$$
\frac{\partial Q_{13}}{\partial x_{1}}+\frac{\partial Q_{23}}{\partial x_{2}}+2 \tilde{q}_{3}=\rho H \ddot{r} .
$$

Bending and torsion moments are determined in the form:

$$
\begin{gathered}
M_{11}=\int_{-h}^{h} \sigma_{11} z \mathrm{~d} z=\frac{H^{2}}{2}\left(E_{11} \frac{\partial \tilde{\psi}_{1}}{\partial x_{1}}+E_{12} \frac{\partial \tilde{\psi}_{2}}{\partial x_{2}}-E_{13} \frac{2(\tilde{r}-\tilde{W})}{H}\right), \\
M_{22}=\int_{-h}^{h} \sigma_{22} z \mathrm{~d} z=\frac{H^{2}}{2}\left(E_{12} \frac{\partial \tilde{\psi}_{1}}{\partial x_{1}}+E_{22} \frac{\partial \tilde{\psi}_{2}}{\partial x_{2}}-E_{23} \frac{2(\tilde{r}-\tilde{W})}{H}\right), \\
M_{12}=M_{21}=\int_{-h}^{h} \sigma_{12} z \mathrm{~d} z=G_{12} \frac{H^{2}}{2}\left(\frac{\partial \tilde{\psi}_{1}}{\partial x_{2}}+\frac{\partial \tilde{\psi}_{2}}{\partial x_{1}}\right) .
\end{gathered}
$$

Expressions for crosscutting forces have the form:

$$
Q_{13}=\int_{-h}^{h} \sigma_{13} \mathrm{~d} z=G_{13}\left(2 \tilde{u}_{1}+H \frac{\partial \tilde{r}}{\partial x_{1}}\right), \quad Q_{23}=\int_{-h}^{h} \sigma_{23} \mathrm{~d} z=G_{23}\left(2 \tilde{u}_{2}+H \frac{\partial \tilde{r}}{\partial x_{2}}\right) .
$$

In Equations (15) terms with external load are determined by the following formula:

$$
\tilde{q}_{k}=\frac{q_{k}^{(+)}+q_{k}^{(-)}}{2},(k=1,2), \quad \tilde{q}_{3}=\frac{q_{3}^{(+)}-q_{3}^{(-)}}{2} .
$$

To derive other equations we will introduce the following bimoments, generated at bending and shear of the plate. Bimoments $P_{11}, P_{22}, P_{12}$ are determined by the following formula:

$$
\begin{gathered}
P_{11}=\frac{1}{h^{2}} \int_{-h}^{h} \sigma_{11} z^{3} \mathrm{~d} z=\frac{H^{2}}{2}\left(E_{11} \frac{\partial \tilde{\beta}_{1}}{\partial x_{1}}+E_{12} \frac{\partial \tilde{\beta}_{2}}{\partial x_{2}}-E_{13} \frac{2(3 \tilde{\gamma}-\tilde{W})}{H}\right), \\
P_{22}=\frac{1}{h^{2}} \int_{-h}^{h} \sigma_{22} z^{3} \mathrm{~d} z=\frac{H^{2}}{2}\left(E_{12} \frac{\partial \tilde{\beta}_{1}}{\partial x_{1}}+E_{22} \frac{\partial \tilde{\beta}_{2}}{\partial x_{2}}-E_{23} \frac{2(3 \tilde{\gamma}-\tilde{W})}{H}\right), \\
P_{12}=P_{21}=\frac{1}{h^{2}} \int_{-h}^{h} \sigma_{12} z^{3} \mathrm{~d} z=\frac{H^{2}}{2} G_{12}\left(\frac{\partial \tilde{\beta}_{1}}{\partial x_{2}}+\frac{\partial \tilde{\beta}_{2}}{\partial x_{1}}\right) .
\end{gathered}
$$

Intensity of transversal tangential and normal bimoments $\tilde{p}_{13}, \tilde{p}_{23}$ and $\tilde{p}_{33}$ are determined by expressions

$$
\begin{gathered}
\tilde{p}_{k 3}=\frac{1}{2 h^{3}} \int_{-h}^{h} \sigma_{k 3} z^{2} \mathrm{~d} z=G_{k 3}\left(\frac{2 \tilde{u}_{k}-4 \tilde{\psi}_{k}}{H}+\frac{\partial \tilde{\gamma}}{\partial x_{k}}\right),(k=1,2), \\
\tilde{p}_{33}=\frac{1}{2 h^{2}} \int_{-h}^{h} \sigma_{33} \mathrm{~d} \mathrm{~d} z=E_{31} \frac{\partial \tilde{\psi}_{1}}{\partial x_{1}}+E_{32} \frac{\partial \tilde{\psi}_{2}}{\partial x_{2}}-E_{33} \frac{2(\tilde{r}-\tilde{W})}{H} .
\end{gathered}
$$

Equations relative to bimoments at bending and transversal shear are derived in the form:

$$
\begin{gathered}
\frac{\partial P_{11}}{\partial x_{1}}+\frac{\partial P_{12}}{\partial x_{2}}-3 \tilde{p}_{13}+H \tilde{q}_{1}=\frac{H^{2}}{2} \rho \ddot{\tilde{\beta}}_{1}, \quad \frac{\partial P_{21}}{\partial x_{1}}+\frac{\partial P_{22}}{\partial x_{2}}-3 \tilde{p}_{23}+H \tilde{q}_{2}=\frac{H^{2}}{2} \rho \ddot{\tilde{\beta}}_{2}, \\
H \frac{\partial \tilde{p}_{13}}{\partial x_{1}}+H \frac{\partial \tilde{p}_{23}}{\partial x_{2}}-4 \tilde{p}_{33}+2 \tilde{q}_{3}=H \rho \ddot{\tilde{\gamma}} .
\end{gathered}
$$

From expressions for series (4) and Formulas (14) relations for series' coefficients $B_{2 i+1}^{(1)}, B_{2 i+1}^{(2)}, A_{2 i},(i=0,1,2)$ (4) via $\tilde{u}_{k}, \tilde{\psi}_{k}, \tilde{\beta}_{k}$ functions and boundary conditions (3) lets represent like next equations: 


$$
\begin{aligned}
& \tilde{u}_{k}=\frac{1}{2}\left(21 \tilde{\beta}_{k}-7 \tilde{\psi}_{k}\right)-\frac{1}{30} H \frac{\partial \tilde{W}}{\partial x_{k}}+\frac{1}{30} \frac{H \tilde{q}_{k}}{G_{k 3}}, \quad(k=1,2), \\
& \tilde{W}=\frac{1}{4}(21 \tilde{\gamma}-3 \tilde{r})-\frac{1}{20} H\left(\frac{E_{31}}{E_{33}} \frac{\partial \tilde{u}_{1}}{\partial x_{1}}+\frac{E_{32}}{E_{33}} \frac{\partial \tilde{u}_{2}}{\partial x_{2}}\right)+\frac{H \tilde{q}_{3}}{20 E_{33}} .
\end{aligned}
$$

The system of differential equations of motion (15), (20), (21) and (22) makes combined system of nine equations relative to nine unknown functions $\tilde{\psi}_{1}, \tilde{\psi}_{2}, \tilde{u}_{1}, \tilde{u}_{2}, \tilde{\beta}_{1}, \tilde{\beta}_{1}, \tilde{r}, \tilde{\gamma}, \tilde{W}$.

Formula to determine the displacements and stresses in the layers of the plate $z=-h$ and $z=h$ are:

$$
\begin{aligned}
& u_{i}^{(-)}=\bar{u}_{i}-\tilde{u}_{i}, \quad u_{i}^{(+)}=\bar{u}_{i}+\tilde{u}_{i}, \quad(i=1,2), \quad u_{3}^{(-)}=\tilde{W}-\bar{W}, \quad u_{3}^{(+)}=\tilde{W}+\bar{W}, \\
& \sigma_{i j}^{(-)}=\bar{\sigma}_{i j}-\tilde{\sigma}_{i j}, \quad \sigma_{i j}^{(+)}=\bar{\sigma}_{i j}+\tilde{\sigma}_{i j}, \quad(i=1,2 ; j=1,2) .
\end{aligned}
$$

Thus, two unrelated problems of bimoment theory of thick plates are formulated in the paper. An accuracy of bimoment theory is defined in dependence on the number of held terms of the series (4). In construction of equations of equilibrium eight terms are held, while for expressions (13) and (22) six terms of each series are held (4). The first equation in (13) and the second equation in (22) are built up to the fourth order relative to small parameter of the plate $\frac{H}{10 a}$. The second Equation in (13) and the first Equation in (22) are built up to the sixth order relative to the parameter.

\section{Solution of Tests Problem}

As an example consider the problem of static bending of the plate, loaded by normal load:

$q_{3}^{(-)}=-q_{0} \sin \frac{\pi x_{1}}{a} \sin \frac{\pi x_{2}}{b}$ along the upper face surface $z=-h$, where $q_{0}$-is a load parameter. Let the ends of the plate rest on the ends, then $x_{1}=$ const and $x_{2}=$ const, and we have the conditions :

$$
\begin{aligned}
& N_{11}=0, T_{11}=0, \bar{\psi}_{2}=0, \bar{\beta}_{2}=0, \bar{\sigma}_{11}=0, \bar{u}_{2}=0, \bar{r}=0, \bar{\gamma}=0, \bar{W}=0, \\
& N_{22}=0, T_{22}=0, \quad \bar{\psi}_{1}=0, \bar{\beta}_{1}=0, \bar{\sigma}_{22}=0, \bar{u}_{1}=0, \bar{r}=0, \bar{\gamma}=0, \bar{W}=0, \\
& M_{11}=0, \quad P_{11}=0, \quad \tilde{\psi}_{2}=0, \quad \tilde{\beta}_{2}=0, \quad \tilde{\sigma}_{11}=0, \quad \tilde{u}_{2}=0, \quad \tilde{r}=0, \quad \tilde{\gamma}=0, \quad \tilde{W}=0, \\
& M_{22}=0, \quad P_{22}=0, \quad \tilde{\psi}_{1}=0, \quad \tilde{\beta}_{1}=0, \quad \tilde{\sigma}_{22}=0, \quad \tilde{u}_{1}=0, \quad \tilde{r}=0, \quad \tilde{\gamma}=0, \quad \tilde{W}=0 .
\end{aligned}
$$

The values $\bar{\sigma}_{11}, \bar{\sigma}_{22}, \bar{\sigma}_{12}, \tilde{\sigma}_{11}, \tilde{\sigma}_{12}, \tilde{\sigma}_{22}$ are determined by Hooke's law with conditions on face surfaces (3):

$$
\begin{aligned}
& \bar{\sigma}_{11}=E_{11}^{*} \frac{\partial \bar{u}_{1}}{\partial x_{1}}+E_{12}^{*} \frac{\partial \bar{u}_{2}}{\partial x_{2}}+\frac{E_{13}}{E_{33}} \bar{q}_{3}, \quad \bar{\sigma}_{22}=E_{12}^{*} \frac{\partial \bar{u}_{1}}{\partial x_{1}}+E_{22}^{*} \frac{\partial \bar{u}_{2}}{\partial x_{2}}+\frac{E_{23}}{E_{33}} \bar{q}_{3}, \quad \bar{\sigma}_{12}=G_{12}\left(\frac{\partial \bar{u}_{1}}{\partial x_{2}}+\frac{\partial \bar{u}_{2}}{\partial x_{1}}\right), \\
& \tilde{\sigma}_{11}=E_{11}^{*} \frac{\partial \tilde{u}_{1}}{\partial x_{1}}+E_{12}^{*} \frac{\partial \tilde{u}_{2}}{\partial x_{2}}+\frac{E_{13}}{E_{33}} \tilde{q}_{3}, \quad \tilde{\sigma}_{22}=E_{12}^{*} \frac{\partial \tilde{u}_{1}}{\partial x_{1}}+E_{22}^{*} \frac{\partial \tilde{u}_{2}}{\partial x_{2}}+\frac{E_{23}}{E_{33}} \tilde{q}_{3}, \quad \tilde{\sigma}_{12}=G_{12}\left(\frac{\partial \tilde{u}_{1}}{\partial x_{2}}+\frac{\partial \tilde{u}_{2}}{\partial x_{1}}\right),
\end{aligned}
$$

here $E_{11}^{*}=E_{11}-\frac{E_{13}}{E_{33}} E_{31}, E_{22}^{*}=E_{22}-\frac{E_{23}}{E_{33}} E_{32}, E_{12}^{*}=E_{12}-\frac{E_{23}}{E_{33}} E_{31}$.

Solution of the Equations (6), (11), (12) and (13), satisfying boundary conditions (24), is written in the form:

$$
\begin{aligned}
& \bar{\psi}_{1}=\bar{C}_{1} f_{1}\left(x_{1}, x_{2}\right), \bar{\psi}_{2}=\bar{C}_{2} f_{2}\left(x_{1}, x_{2}\right), \bar{r}=\bar{C}_{3} f_{3}\left(x_{1}, x_{2}\right), \bar{\beta}_{1}=\bar{C}_{4} f_{1}\left(x_{1}, x_{2}\right), \bar{\beta}_{2}=\bar{C}_{5} f_{2}\left(x_{1}, x_{2}\right), \\
& \bar{u}_{1}=\bar{C}_{6} f_{1}\left(x_{1}, x_{2}\right), \bar{u}_{2}=\bar{C}_{7} f_{2}\left(x_{1}, x_{2}\right), \bar{W}=\bar{C}_{8} f_{3}\left(x_{1}, x_{2}\right), \bar{\gamma}=\bar{C}_{9} f_{3}\left(x_{1}, x_{2}\right),
\end{aligned}
$$

where $f_{1}\left(x_{1}, x_{2}\right)=\cos \left(\frac{\pi x_{1}}{a}\right) \sin \left(\frac{\pi x_{2}}{b}\right), f_{2}\left(x_{1}, x_{2}\right)=\sin \left(\frac{\pi x_{1}}{a}\right) \cos \left(\frac{\pi x_{2}}{b}\right), f_{3}\left(x_{1}, x_{2}\right)=\sin \left(\frac{\pi x_{1}}{a}\right) \sin \left(\frac{\pi x_{2}}{b}\right)$.

Solution of the Equations (15), (20), (21) and (22), satisfying boundary conditions (24), has the form: 


$$
\begin{array}{ll}
\tilde{\psi}_{1}=\tilde{C}_{1} f_{1}\left(x_{1}, x_{2}\right), \quad \tilde{\psi}_{2}=\tilde{C}_{2} f_{2}\left(x_{1}, x_{2}\right), \quad \tilde{r}=\tilde{C}_{3} f_{3}\left(x_{1}, x_{2}\right), \quad \tilde{\beta}_{1}=\tilde{C}_{4} f_{1}\left(x_{1}, x_{2}\right), \quad \tilde{\beta}_{2}=\tilde{C}_{5} f_{2}\left(x_{1}, x_{2}\right), \\
\tilde{u}_{1}=\tilde{C}_{6} f_{1}\left(x_{1}, x_{2}\right), \quad \tilde{u}_{2}=\tilde{C}_{7} f_{2}\left(x_{1}, x_{2}\right), \quad \tilde{W}=\tilde{C}_{8} f_{3}\left(x_{1}, x_{2}\right), \quad \tilde{\gamma}=\tilde{C}_{9} f_{3}\left(x_{1}, x_{2}\right) .
\end{array}
$$

Substituting solution (27) into Equations (6), (11), (12) (13), we will obtain the system of linear algebraic equations relative to nine unknown constants $C_{1}, C_{2}, \cdots, C_{9}$. In similar way, substituting solution (28) into Equations (15), (20), (21), (22), we will obtain one more system of linear algebraic equations relative to nine unknown constants.

Analysis for orthotropic square plate with elastic characteristics is conducted [1]:

$$
E_{1}=4.6 \times E_{0}, E_{2}=1.6 \times E_{0}, E_{1}=1.12 \times E_{0} ; G_{12}=0.56 \times E_{0}, G_{13}=0.43 \times E_{0}, G_{23}=0.33 \times E_{0},
$$

where $E_{0}=10^{4} \mathrm{MPa} ; v_{21}=0.27, v_{23}=0.3, v_{31}=0.07$.

Table 1 and Table 2 give dimensionless numerical results of calculations of displacements and stresses in upper and lower layers of the plate. Maximal values of displacements and stresses of the plate are reached in face surfaces of the pate and are determined by solutions of the first and the second problems.

Table 3 and Table 4 give dimensionless numerical results of displacements and stresses in the middle surface. It should be noted that coefficients of the series with zero indices are displacements $B_{0}^{(1)}=u_{1}^{(0)}, B_{0}^{(2)}=u_{2}^{(0)}, A_{0}=u_{3}^{(0)}$ and kinematic functions $B_{1}^{(1)}, B_{1}^{(2)}, A_{1}$, characterize rotary angle of normal vector and strain of transversal compression of middle surface of the plate.

Analysis has shown that the values of normal displacement $u_{3}$ vary considerably along the thickness of the plate. In [4] [8] it is shown that maximal values of stresses and displacements, obtained according to bimoment theory for isotropic plates with high accuracy agree with calculations of an exact solution [4].

Table 1. Values of displacements and stresses in the upper layer of the plate.

\begin{tabular}{ccccccc}
\hline$H / b$ & $E_{0} u_{1}^{(-)} / H q_{0}$ & $E_{0} u_{2}^{(-)} / H q_{0}$ & $E_{0} u_{3}^{(-)} / H q_{0}$ & $\sigma_{11}^{(-)} / q_{0}$ & $\sigma_{22}^{(-)} / q_{0}$ & $\sigma_{12}^{(-)} / q_{0}$ \\
\hline $1 / 3$ & 0.5282 & 0.7210 & 2.6566 & -2.9471 & -1.5908 & 0.7326 \\
$1 / 4$ & 1.1970 & 1.5193 & 5.8987 & -4.7690 & -2.2905 & 1.1947 \\
$1 / 5$ & 2.3131 & 2.7671 & 11.7869 & -7.1799 & -3.1620 & 1.7875 \\
$1 / 6$ & 3.9885 & 4.5727 & 21.5969 & -10.1601 & -4.2010 & 2.5103 \\
$1 / 10$ & 18.5117 & 19.5965 & 135.5503 & -27.6397 & -10.2253 & 6.7043 \\
\hline
\end{tabular}

Table 2. Values of displacements and stresses in the lower layer of the plate.

\begin{tabular}{ccccccc}
\hline$H / b$ & $E_{0} u_{1}^{(+)} / H q_{0}$ & $E_{0} u_{2}^{(+)} / H q_{0}$ & $E_{0} u_{3}^{(+)} / H q_{0}$ & $\sigma_{11}^{(+)} / q_{0}$ & $\sigma_{22}^{(+)} / q_{0}$ & $\sigma_{12}^{(+)} / q_{0}$ \\
\hline $1 / 3$ & -0.5119 & -0.7871 & 2.2516 & 2.5562 & 1.3123 & -0.7618 \\
$1 / 4$ & -1.2028 & -1.6414 & 5.4911 & 4.4799 & 2.0546 & -1.2509 \\
$1 / 5$ & -2.3388 & -2.9410 & 11.3784 & 6.9456 & 2.9474 & -1.8578 \\
$1 / 6$ & -4.0325 & -4.7958 & 21.1880 & 9.9581 & 4.0074 & -2.5886 \\
$1 / 10$ & -18.6193 & -20.0046 & 135.1412 & 27.4884 & 10.0410 & -6.7951 \\
\hline
\end{tabular}

Table 3. Values of displacements and rotary angle of a normal in middle surface of the plate.

\begin{tabular}{cccccc}
\hline$H / b$ & $E_{0} B_{0}^{(1)} / H q_{0}$ & $E_{0} B_{0}^{(2)} / H q_{0}$ & $E_{0} A_{1} / H q_{0}$ & $E_{0} B_{1}^{(1)} / H q_{0}$ & $E_{0} B_{1}^{(2)} / H q_{0} A_{0} / H q_{0}$ \\
\hline $1 / 3$ & -0.0296 & -0.0773 & -0.2054 & -0.2079 & -0.5186 \\
$1 / 4$ & -0.0355 & -0.0970 & -0.2062 & -0.7391 & -1.2758 \\
$1 / 5$ & -0.0409 & -0.1168 & -0.2061 & -1.7176 & -2.4808 \\
$1 / 6$ & -0.0463 & -0.1370 & -0.2058 & -3.2571 & -4.2421 \\
$1 / 10$ & -0.0693 & -0.2198 & -0.2051 & -17.2440 & -19.0810 \\
\hline
\end{tabular}


Table 4. Values of stresses in middle surface of the plate.

\begin{tabular}{ccccccc}
\hline$H / b$ & $\sigma_{13}^{(0)} / q_{0}$ & $\sigma_{23}^{(0)} / q_{0}$ & $\sigma_{33}^{(0)} / q_{0}$ & $\sigma_{12}^{(0)} / q_{0}$ & $\sigma_{11}^{(0)} / q_{0}$ & $\sigma_{22}^{(0)} / q_{0}$ \\
\hline $1 / 3$ & 0.7033 & 0.6492 & -0.4904 & -0.0627 & 0.0089 & -0.0438 \\
$1 / 4$ & 1.0019 & 1.0019 & -0.4965 & -0.0582 & -0.0093 & -0.0552 \\
$1 / 5$ & 1.2986 & 1.0357 & -0.4985 & -0.0555 & -0.0210 & -0.0612 \\
$1 / 6$ & 1.5921 & 1.2274 & -0.4992 & -0.0537 & -0.0285 & -0.0647 \\
$1 / 10$ & 2.7469 & 1.9995 & -0.4999 & -0.0509 & -0.0410 & -0.0702 \\
\hline
\end{tabular}

\section{Conclusion}

So on the basis of expansion method, a theory of plates is improved by consideration of bimoments. In the case of spatial deformation of the plate along its thickness, there nonlinear laws of displacements distribution occur, without any simplifying hypotheses. Consequently, existing specified theories of plates and shells, built with a number of simplifying hypotheses could not be used in development of methods of calculation of stresses and displacements of thick plates and shells under the effect of various types of external influences. Calculations of thick plates from anisotropic materials with low strength characteristics could not be made on the basis of classical or specified existing theory. In such cases it is advisable to conduct calculations based on rigorous methodologies developed on the basis of the theory of plates and shells, which takes into account all the components of stress and strain tensor $\sigma_{i j}, \varepsilon_{i j},(i, j=1,3)$.

\section{References}

[1] Ambartsumyan, S.A. (1987) Theory of Anisotropic Plates. Nauka, Moscow, 360 p.

[2] Galimov, Sh.K. (1976) Specified Theories of Design of Rectangular Theory of Orthotropic Plate under Transversal Load. Studies on the Theory of Plates and Shells, Collection of Papers, Issue XII, Kazan, 78-84.

[3] Vlasov, V.Z. (1958) Thin-Walled Spatial System. Gosstroyizdat, Moscow, 503 p.

[4] Usarov, M.K. (2011) Problem of Bending of Thick Orthotropic Plate in Three-Dimensional Statement. EngineeringConstruction Journal, 4, 40-47.

[5] Usarov, M.K. (2014) Theory of Thick Plates with Consideration of Bimoments. Scientific-Technical Journal of FerPI, 3, 44-50.

[6] Usarov, M.K. (2014) Analysis of Thick Orthotropic Plates on the Basis of Bimoment Theory. Uzbek Journal of Problems in Mechanics, 2, 41-44.

[7] Usarov, M.K. (2014) Analysis of Orthotropic Plates on the Basis of Bimoment Theory. Uzbek Journal of Problems in Mechanics, 3-4, 37-41.

[8] Usarov, M.K. (2014) Bimoment Theory of Bending and Vibrations of Thick Orthotropic Plates. Reports of National University of Uzbekistan, 2/1, 127-132. 